\title{
A Two-Stage Buccal Mucosal Graft (BMG) for Managing Recurrent Proximal Penile Hypospadias in Pediatric \& Adolescent Populations
}

\author{
M. G. Elsheikh, A. Fayad \\ Cairo University, Cairo, Egypt \\ E-mail: mohamed.galalelsheikh@gmail.com, mohamed.galalelsheikh@gmail.com \\ Received December 31, 2010; revised February 13, 2011; accepted February 20, 2011
}

\begin{abstract}
Introduction: The presence of a recurrent proximal penile hypospadius represents a surgical challenge to the urologist due to the presence of excessive scarring and fibrosis of the tissues. This problem is more pronounced in circumcised patients, in whom there is no enough skin for one stage procedures. Buccal mucosal grafts represent a good surgical option. The aim of this study was to evaluate the results of two stages buccal mucosal urethroplasty in pediatric \& adolescent patients, presenting with recurrent proximal penile hypospadias who are circumcised. Methods: Thirty seven pediatric \& adolescent patients underwent two stages buccal mucosal urethroplasty for recurrent proximal penile hypospadius. In all cases the buccal graft was placed dorsally followed by the second stage closure after 6 months. Results: The mean age was 17.7 (14-20) years. With a mean follow-up of 28.3 months, 33 patients (89.2\%) had a final successful outcome. Of the 4 cases that were considered as failure, 3 patients (8.1\%) developed urethra-cutaneous fistula that required closure after 3 months. The remaining patient developed meatal stenosis. Conclusion: Although buccal mucosal urethroplasty is a two staged procedure, it is feasible option for pediatric \& adolescent patients presenting with recurrent proximal penile hypospadius, who had no skin available for penile flaps, with a success rate approaching $89.2 \%$.
\end{abstract}

Keywords: Buccal Graft Urethroplasty, Hypospadias

\section{Introduction}

Hypospadias is a congenital abnormality occurring in 1 of 300 live births (1). Nowadays, most cases of hypospadias can be successfully repaired with a one-stage procedure in the first year of life with a success rate approaching $90 \%$ (2). The penile skin with the underlying dartos fascia provides together with the prepuce in uncircumcised children good tissue for primary repair together with managing any encountered complications.

Unfortunately, the repeated attempts at surgical repair in recurrent failures after hypospadias repair would result in densely scarred, immobile, hypovascular, or significantly shortened penis (3).

The repair of these penile hypospadias represents a challenge for the urologists, the tissues being fibrosed, hypovascular and immobile. Furthermore, there is no enough healthy penile skin for repair.

\section{Patients and Methods}

This is a prospective study carried out between February
2006 and April 2009 on 37 pediatric \& adolescent patients presenting with recurrent proximal penile hypospadias. The age of patients ranged between 14 and 20 years (mean 17.7). All patients had undergone a variety of primary hypospadias corrections in the past while 10 patients had two trials of previous repair. All patients were found to be circumcised at time of presentations. Thirty patients had penile chordee.

The main complaint was proximal penile hypospadias, with ventral chordee in 30 patients. The urethral plate was found to be fibrosed and scarred. All patients included in the study were found to be circumcised.

Preoperatively, all patients underwent physical examination to exclude BXO, urine analysis and culture. All our patients and their relatives were informed about the procedure and signed an appropriate consent.

All of the patients were managed by 2 stages buccal mucosal urethroplasty. At the first stage, we performed a urethroscopy under general anesthesia to assess the urethra, then while the patient in the lithotomy position, all the scarred urethral plate was completely removed through a two para-urethral incision that extends to the tip 
of the penis splitting the glans into two wings. Proximally the incisions surround the meatus. All the scarred tissues are excised to straighten the penis. The meatus was accordingly transferred to a more proximal position.

The defect was accurately measured. The defect length ranged between $3.5 \mathrm{~cm}$ to $7.5 \mathrm{~cm}$ with a mean of $5.2 \mathrm{~cm}$.

At that stage, we shifted to the oral cavity for harvesting the buccal graft. A buccal mucosal graft of a relatively the same length and the largest possible width was properly measured and then harvested from the inner side of the cheek taking care to avoid the opening of the parotid duct opposite to the upper 2nd molar. We needed to shift to the other cheek in 5 patients to harvest an additional length. The use of nasal endotracheal tube made the exposure of the oral cavity easier giving a wider space for working. We used to inject diluted epinephrine $(1: 200,000)$ submucosally as this made the harvesting of the graft easier and less bloody. The graft was then transferred to a side table where complete de-fating was carried out. The donor site was left without approximating sutures; any bleeding was controlled with diathermy.

The graft was then transferred to the penis and fixed to the underlying corpora as a dorsal graft with 5-0 vicryl sutures. Multiple sutures were used to properly anchor the graft to the underlying corpora. The edges of the graft were sutured to the dartos fascia and not only the skin to facilitate the later mobilization of the edges for later closure at the time of the second stage. The width of the graft harvested should not be less than $1.5 \mathrm{~cm}$.

A $16 \mathrm{~F}$ silicon catheter was used in 22 patients, while a $14 \mathrm{~F}$ catheter was used in the remaining 15 patients who were of younger age. The catheter was left for one week. The dressing was removed after 3 days and daily dressing with antiseptic solution was carried out 3 times daily. The donor site was cleansed with antiseptic mouth wash and topical anesthetics for the first 3 days.

The second stage was performed after 6 months, to ensure proper vascularisation of the graft. Preoperative urine analysis and culture were performed to ensure sterile urine and prophylactic antibiotics were started preoperatively. Two vertical Para-mucosal incisions were made that allowed the free mobilization of the graft edges to be approximated in the midline without tension. Vicryl sutures (5-0) were used for the closure of the neo-urethra around the silicone catheter. We used $16 \mathrm{~F}$ silicone catheter in 20 patients while $14 \mathrm{~F}$ catheter was used in the remaining 17 cases. The idea of selecting the catheter caliber was to close the graft without any tension. The catheter was left indwelling for 3 weeks.

The mean follow up was 28.3 (12-50) months. Success was defined as having a functioning urethra without fistula, meatal stenosis, stricture or residual chordee and having a cosmetically acceptable glandular meatus. The

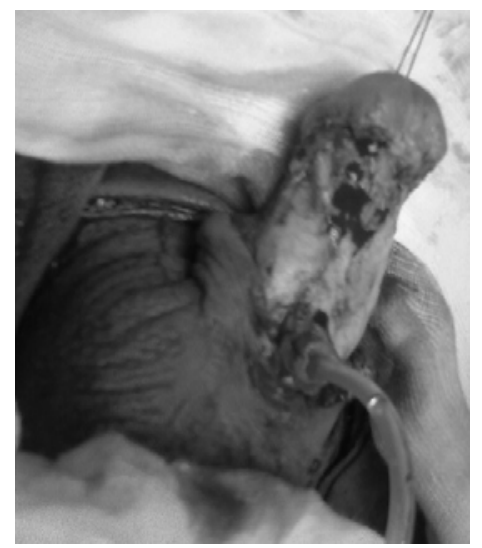

Figure 1

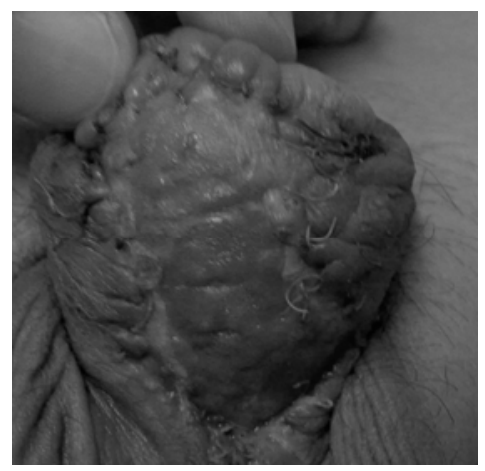

Figure 2

necessity of meatal or urethral dilatation and the presence of complications or a poor aesthetic result requiring revision were considered as failures.

\section{Results}

Our study was carried out on 37 pediatric \& adolescent patients, the mean age was 17.7 (14-20) years. As regard the previous attempts at repair: Snod Grass was tried in 32 cases while transverse preprutial island flap was tried in three cases and longitudinal ventral penile skin flap in the remaining 2 cases. All the ten cases with two trials of repair had Snod Grass being the second procedure.

As regard the first stage; the overall operative time ranged between $125 \mathrm{~min}$ and $210 \mathrm{~min}$ with a mean of 147 . Mean time for harvesting buccal graft was $17 \mathrm{~min}$. The hospital stay ranged between 3-5 days with a mean of 3.4. The dressing was removed on the 3rd post-operative day. 5 of our patients had their graft being infected and needed swabs from the graft for culture and sensitivity. The antibiotic was prescribed for a week. None of our patients showed graft loss after their first stage.

As regard the 2nd stage; the overall operative time ranged between 105min and 180min with a mean of 135 . The hospital stay ranged between 2-4 days with a mean of 
3.16.

Of the 37 cases included in the study, 33 (89.2\%) were classified as successes and 4 cases $(10.8 \%)$ as failures. The failures were due to urethro-cutaneous fistula in 3 cases (8.1\%) and meatal stenosis in one case (2.7\%).

The fistula developed immediately after removal of urethral catheter after the second stage. The patient was considered as failure and underwent closure of the fistula successfully 3 months later. Meatal stenosis was the other cause of failure; the patient was 18 years old, and presented with gradual decrease in the urinary flow after removal of the urethral catheter after successful 2nd stage. He needed meatal dilatation for 2 weeks thereafter. None of the patients who were considered as success needed any further intervention during the follow-up. Furthermore, none of our patients experienced any degree of penile chordee. Also, no one complained from any oral problems during the follow-up.

\section{Discussion}

The management of complications in pediatric and adolescent age groups, in whom multiple attempts of hypospadias repair had failed, is a surgical challenge and still represents a complex problem for reconstructive urologists. This is attributed to the fact that repeated attempts at repair would result in tissues that are fibrosed, hypovascular and immobile. The deficiency of enough unscarred penile skin, being used for previous attempt at repair, adds another difficulty.

Historically, various tissues have been used to repair the damaged urethra including genital (penile and scrotal) skin, extra-genital skin, bladder mucosa and buccal mucosa. These tissues have been used as either pedicled flaps with their own blood supply or as free tissue grafts. The most common graft materials in use today are buccal mucosa, preputial skin (when available) and penile and preputial skin flaps with their own blood supply.

The use of buccal mucosa (BM) in urethral surgery was first described by Humby in 1941, (4) however, it was not reported again until the late 1980s.

The main reported advantages of the buccal mucosa that favors its use in substitution urethroplasty is it's nature being wet epithelium, it is readily harvested, it has been shown to be resistant to recurrence of strictures (especially in the presence of balanitis xerotica obliterans, $\mathrm{BXO}$ ). The presence of a dense submucosa with a dense capillary network facilitates early imbibition and early inosculation (5).

It was not until 1992 when Burger et al. reported the first use of BM for repair of complications following childhood hypospadias surgery (6).

In 1995, Bracka conducted his study on managing complications after hypospadias repair and he concluded that a two-stage repair by splitting the glans and lining it with penile skin or BM grafts was extremely adaptable and successful (7).

In studying the outcomes of buccal mucosa (BM) penile urethroplasty, most of the studies reported a high success rate (8).

In 1998 Mundy et al reported a success rate of $100 \%$ on 8 cases, as 2 stages buccal mucosal urethroplasty due to penile stricture caused by BXO (9). Three years later, he published his results on 39 cases underwent 2 stages procedures and followed -up for at least 2 years and reported a success of $83 \%$ (5).

Dubey et al reported a series of 43 patients undergoing dorsal-onlay BM urethroplasty for penile strictures. 28 of their patients had a one-stage procedure, while 15 of their patients required two-stages. The mean follow-up was shorter for two-stage reconstruction (24.2 months) compared to one-stage procedures (34 months). They reported success rate of $86.7 \%$ for two-stage procedures, with the majority of recurrences being managed successfully with DVIU (10). The same authors reported the outcomes of the 2 stage procedure on 14 cases with strictures due to $\mathrm{BXO}$, after a mean follow-up of 32.5 months, they reported a success rate of $78.6 \%$ (11).

Our success rate was $89.2 \%$, a result which is comparable to that published by Mundy et al (5) and Dubey et al in his first series (10) but much better than that reported by the same author in his second series (11).This might be attributed to the fact that most published series on two stages buccal urethroplasty was done for BXO, this is not the case in our study as none of our patients had BXO.

In our study, none of our patients developed contracture of the initial graft, and none of the succeeded cases required more than the two previously planned surgeries. That's why we do not agree with Shukla et al. in 2004 (12), who concluded his study that a 2-stage repair is actually a misnomer and that about $70 \%$ of cases required at least one additional procedure (a mean of extra 1.6 procedures).

\section{Conclusion}

Although, there is no single technique that can fit for all situations, the use of 2 stage buccal mucosal urethroplasty appears to be a successful and feasible option for management of patients with recurrent proximal penile hypospadias in the absence of sufficient penile skin with a success rate approaching $89.2 \%$. Although most of our patients have been followed up for more than 2 years yet, longer follow-up is still required.

\section{References}

[1] L. S. Baskin, "Hypospadias and Urethral Development", 
Journal of Urology, Vol. 163, 2000, pp. 951-956.

[2] G. Manzoni, A. Braccka, E. Palminteri and G. Marrocco, "Hypospadias Surgery: When, What and by Whom?” BJU International, Vol. 94, 2004, pp. 1188-1195.

[3] J. F. Stecker, C. E. Horton, C. J. Devine and J. B. McCraw, "Hypospadias Cripples," Urologic Clinics North Amemrica, Vol. 8, 1981, pp. 539-544.

[4] G. Humby, "A One-Stage Operation for Hypospadias," British of Journal Surgery, Vol. 29, 1941, p. 84.

[5] D. E. Andrich and A. R. Mundy, "Substitution Urethroplasty with Buccal Mucosal-Free Grafts," Journal of Urology, Vol. 65, 2001, p. 1131.

[6] R. A. Burger, S. C. Muller, H. El-Damanhoury, A. Tschakaloff, H. Riedmiller and R. Hohenfellner, "The Buccal Mucosal Graft for Urethral Reconstruction: A Preliminary Report,” Journal of Urology, Vol. 147, 1992, pp. 662-664.

[7] A. Bracka, “A Versatile Two-Stage Hypospadias Repair," British Journal of Plastic Surgery, Vol. 48, 1995, pp. 345-352.
[8] J. M. Patterson and C. R. Chapple, "Surgical Techniques in Substitution Urethroplasty Using Buccal Mucosa for the Treatment of Anterior Urethral Strictures,” European Urology, Vol. 53 (6), 2008, pp. 1162-1171.

[9] S. N. Venn and A. R. Mundy, "Urethroplasty for Balanitis Xerotica Obliterans,” British Journal of Urology, Vol. 81, 1998, p. 735.

[10] D. Dubey, A. Kumar, A. Mandhani, et al., "Buccal Mucosal Urethroplasty: A Versatile Technique for All Urethral Segments,” BJU International, Vol. 95, 2005, p. 625.

[11] D. Dubey, A. Sehgal, A. Srivastava, et al., "Buccal Mucosal Urethroplasty for Balanitis Xerotica Obliterans Related Urethral Strictures: The Outcome of 1 and 2-Stage Techniques,” Journal of Urology, Vol. 179, 2005, p. 463.

[12] A. R. Shukla, R. P. Patel and D. A. Canning, "The 2-Stage Hypospadias Repair. Is It a Misnomer?” Journal of Urology, Vol. 172, 2004, pp. 1714-1716. 